\title{
Glaucoma secundário à fístula carótido-cavernosa espontânea com resolução após a embolização da fístula: relato de caso
}

\author{
Secondary glaucoma following carotid cavernous fistula resolved after \\ fistula's embolization: case report
}

Marcelo Jarczun $K_{a c}{ }^{1}$, Sansão Isaac Kac ${ }^{2}$, Arlete Gonçalves dos Santos Martins ${ }^{3}$

\section{RESUMO}

Fístulas carótido-cavernosas espontâneas são raras e ocorrem mais frequentemente em mulheres de meia-idade. Os autores relatam um caso de uma fístula carótido-cavernosa espontânea em mulher idosa evoluindo com glaucoma de difícil controle. Após a embolização cirúrgica da fístula a paciente apresentou regressão da sintomatologia e normalização da pressão intraocular.

Descritores: Fístula carótido-cavernosa; Glaucoma; Pressão intraocular; Angiografia; Relatos de casos

\begin{abstract}
Spontaneous carotid cavernous fistulas are a rare entity occurring more frequently in middle aged women. The authors report a case of a spontaneous carotid cavernous fistula in an elderly woman resulting in glaucoma of hard management. After surgicalembolization of the fistula, the symptoms regressed and the intraocular pressure moved back to regular levels.
\end{abstract}

Keywords: Carotid-cavernous sinus fistula; Glaucoma; Intraocular pressure Angiography; Case reports

\section{INTRODUÇÃO}

- ístulas carótido-cavernosas consistem em uma comunica- ção anormal entre o sistema arterial carotídeo e o seio cavernoso venoso ${ }^{(1-2)}$. Elas surgem após trauma ou de modo espontâneo(1). A mistura de sangue arterial e venoso leva a um aumento da pressão venosa episcleral, ocasionando na maioria dos pacientes um aumento da pressão intraocular $(\mathrm{PIO})^{(3)}$. Essa condição tem sido chamada de "síndrome da fístula dural e do olho vermelho" ou "síndrome da fístula dural"(4).

No presente trabalho relatamos um caso de glaucoma pós trabecular ocasionado por uma fístula carótido-cavernosa espontânea com completa resolução após a embolização cirúrgica da mesma.

\section{RELATO DE CASO}

A.E.S.I., sexo feminino, 81 anos de idade, branca, encaminhada devido à cefaléia há um mês e dor no olho direito há sete dias. Referia hipertensão arterial sistêmica e hipotireoidismo em tratamento. Negava trauma e outras doenças oculares ou sistêmicas.

Ao exame a acuidade visual com correção era de 20/30 no olho direito e 20/25 no olho esquerdo. Exoftalmometria reve-

\footnotetext{
Trabalho realizado no Departamento de Oftalmologia, Universidade Federal Fluminense UFF - Niterói (RJ), Brasil.

${ }^{1}$ Médico, Setor de Glaucoma, Universidade Federal Fluminense - UFF - Niterói (RJ), Brasil. 2 Médico, Setor de Oftalmopediatria, Hospital Servidores do Estado - HSE - Rio de Janeiro (RJ), Brasil.

${ }^{3}$ Médico, Hospital Servidores do Estado - HSE - Rio de Janeiro (RJ), Brasil.

Fontes de auxílio à pesquisa: Não foi utilizada.

Endereço para correspondência: Marcelo Jarczun Kac. Av. Nossa Senhora de Copacabana, 680 - SI. 1.203 - Rio de Janeiro (RJ) - CEP 22050-000

E-mail: marcelojkac@gmail.com

Recebido para publicação em 28.07.2009

Última versão recebida em 20.11.2009

Aprovação em 29.11.2009
}

lou uma proptose do olho direito de $4 \mathrm{~mm}$. A motilidade ocular extrínseca não continha restrições. À biomicroscopia encontramos vasos conjuntivais dilatados e tortuosos na região temporal do olho direito (Figura 1A). A profundidade da câmara anterior estava preservada. A PIO medida com o tonômetro de aplanação de Goldmann foi de $22 \mathrm{mmHg}$ no olho direito e $12 \mathrm{mmHg}$ no olho esquerdo às 18:50 h. Na gonioscopia conseguíamos visualizar esporão escleral $360^{\circ} \mathrm{em}$ ambos os olhos com trabeculado pigmentado 2+/4+. Havia presença de sangue no canal de Schlemm do olho direito. A retinografia demonstrava uma assimetria de escavação dos nervos ópticos, com aumento da escavação no olho direito $(0,6 \times 0,6)$ com discreta redução da rima inferior (Figura $1 C$ ). Já o olho esquerdo possuía uma escavação de 0,4 X 0,4 sem perdas localizadas (Figura 1D).

A ressonância magnética com contraste demonstrou proptose discreta à direita, veia oftálmica superior direita ectasiada e fístula carótido-cavernosa (Figura 2A). Mesmo com o uso de medicação tópica hipotensora (latanoprost 0,005\% colírio 1x ao dia e brinzolamida $1 \%$ colírio $3 x$ ao dia) a $\mathrm{PIO}$ sofreu uma elevação para $34 \mathrm{mmHg}$ no olho direito. O neurologista recomendou a realização de uma arteriografia para embolização da fístula. Na arteriografia constatou-se a presença de uma fístula carótido-cavernosa indireta junto ao seio cavernoso direito, nutrida por ramos durais das artérias carótidas internas e da artéria carótida externa direita (Figura 2B). O exame da carótida esquerda revelou-se normal (Figura 2C). No mesmo ato foi realizado o tratamento endovascular, onde cateterizouse seletivamente a fístula, e foram depositadas micromolas em seu interior associado a um balão destacável. Não houve intercorrências durante este procedimento.

Na consulta após a embolização da fístula a paciente referia melhora da sintomatologia. Na biomicroscopia pudemos evidenciar que os vasos conjuntivais e episclerais do olho direito apresentaram importante redução da dilatação e tortuosidade (Figura 1B). A PIO medida foi de $16 \mathrm{mmHg}$ no olho direito e $12 \mathrm{mmHg}$ no olho esquerdo sem o uso de medicação antiglaucomatosa. Na gonioscopia não era mais possí- 

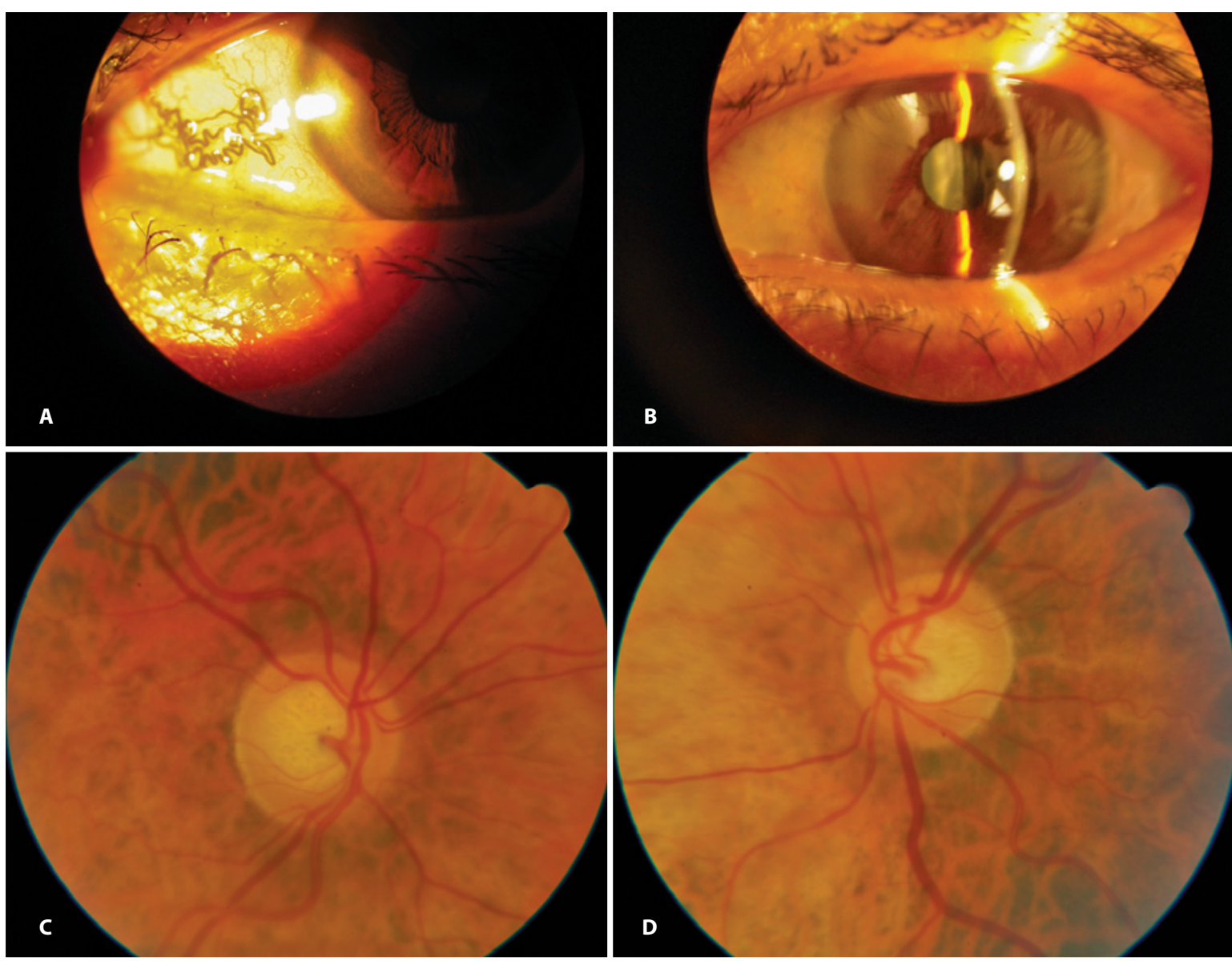

Figura 1. A) Vasos conjuntivais e episclerais do olho direito dilatados e com aumento da tortuosidade; B) Mesmo olho após a embolização da fístula com regressão das alterações citadas; C) Retinografia mostrando escavação de 0,6 X 0,6 com discreta redução da rima inferior no olho direito; D) Retinografia mostrando escavação $0,4 \times 0,4$ no olho esquerdo.

vel visualizar a presença de sangue no canal de Schlemm. Na perimetria automatizada branco no branco realizada antes e após a embolização da fístula, verificamos a manutenção das alterações do campo visual no olho direito (Figura 2D). Vale ressaltar que o índice de confiabilidade é baixo nos dois exames. Já no olho esquerdo obtivemos melhores índices de confiabilidade, e observamos no exame pós embolização uma melhora do campo visual que poderia ser justificada pelo efeito aprendizado (Figura 2E).

\section{COMENTÁRIOS}

Fístulas carótido-cavernosas espontâneas ocorrem mais frequentemente em mulheres de meia-idade ou mais velhas sem história de trauma ${ }^{(3)}$. Nestes casos uma pequena fístula é alimentada por um ramo meníngeo da artéria carótida interna intracavernosa ou da artéria carótida externa, que drena diretamente no seio cavernoso ou na veia dural adjacente que se conecta com o seio cavernoso ${ }^{(4-5)}$

Redução da acuidade visual causada por glaucoma secundário ao aumento da pressão venosa espiscleral é o problema ocular mais frequente ${ }^{(2)}$. Esta condição pode ser de difícil tratamento devido ao fato de que a maioria das medicações antiglaucomatosas apenas reduzem a diferença entre a pres- são intraocular e a pressão venosa episcleral atuando na redução da primeira pela diminuição da produção do humor aquoso ${ }^{(2,6-8)}$. Também podem ser consequências de fístulas: rubeosis, retinopatia isquêmica, oclusão de veia central da retina, oclusão de artéria central da retina, neuropatia óptica, e alterações corneanas secundárias à exposição(2,7-9).

Quando se opta pela intervenção cirúrgica em fístulas durais devemos considerar o risco versus benefício de tal intervenção, o que pode nos conduzir num primeiro momento a escolher o tratamento clínico ${ }^{(3)}$. A intervenção cirúrgica consiste na oclusão por balão intra-arterial ou embolização(2) As taxas de sucesso descritas variam de $58 \%$ a $100 \%{ }^{(2)}$, mas complicações podem ocorrer, incluindo isquemias do segmento anterior, do nervo óptico, e cerebral ${ }^{(7)}$. Devido ao fechamento espontâneo de muitas fístulas, especialmente em casos de síndrome da fístula dural, o tratamento conservador é recomendado em pacientes menos graves, com embolização reservada para aqueles com perda visual ou sinais de piora da condição clínica ${ }^{(10-11)}$.

Neste caso a embolização mostrou-se necessária devido ao glaucoma refratário apresentado pela paciente. Como as condições clínicas eram satisfatórias optou-se pela intervenção cirúrgica que se demonstrou bem sucedida tanto no fechamento da fístula quanto no controle da PIO. 


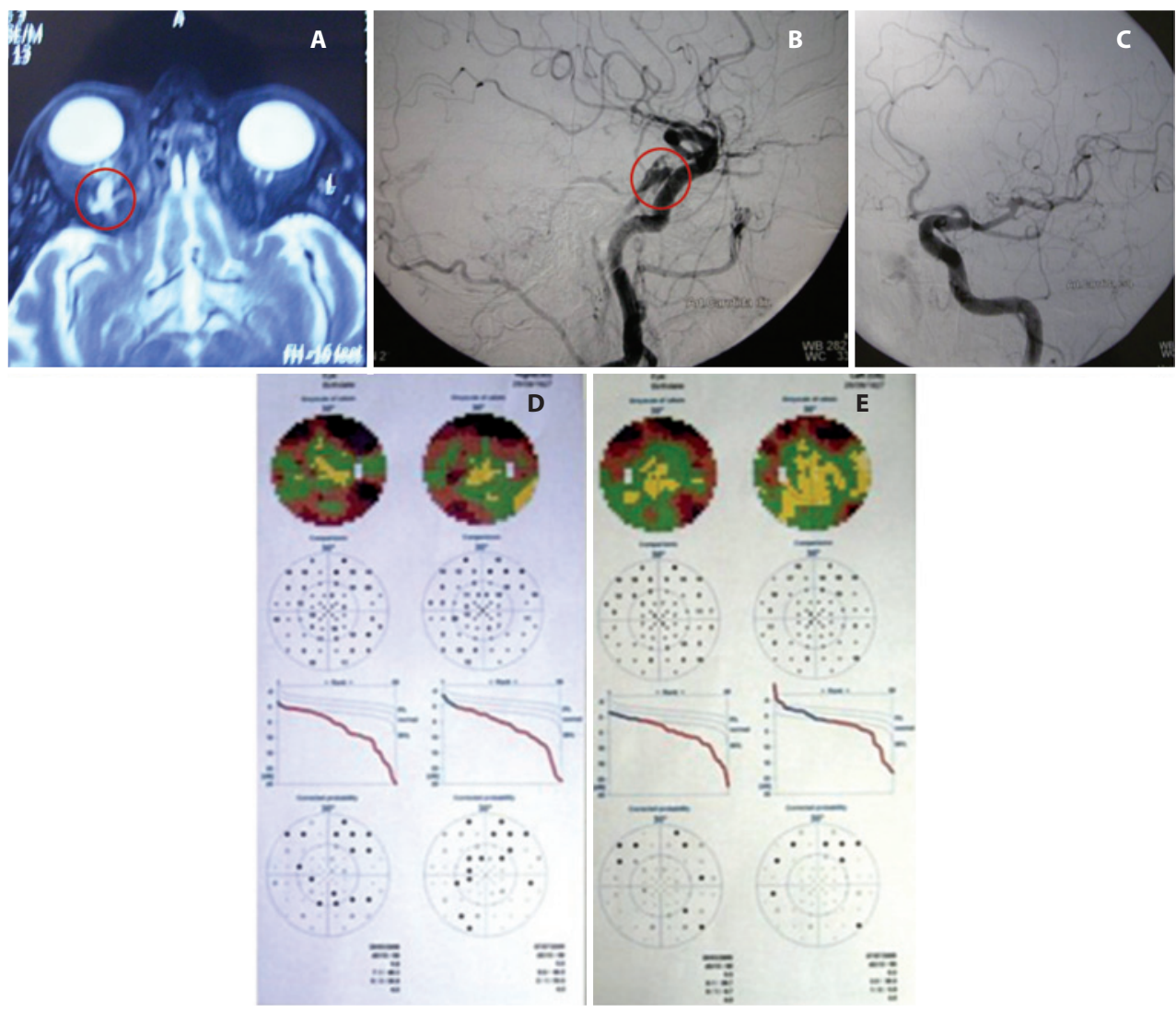

Figura 2. A) Ressonância magnética com contraste evidenciando proptose do olho direito e destaque para a ectasia da veia oftálmica superior direita; B) Arteriografia da carótida direita com local da fístula destacado; C) Arteriografia da carótida esquerda sem alterações; D) Campo visual do olho direito pré e pós embolização da fístula; E) Campo visual do olho esquerdo pré e pós embolização da fístula.

\section{REFERÊNCIAS}

1. Gupta S, Thakur AS, Bhardwaj N, Singh H. Carotid cavernous sinus fistula presenting with pulsating exophthalmos and secondary glaucoma. J Indian Med Assoc. 2008;106(5):312,346

2. Keltner JL, Satterfield D, Dublin AB, Lee BC. Dural and carotid cavernous sinus fistulas. Diagnosis, management, and complications. Ophthalmology. 1987; 94(12):1585-600.

3. Allingham RR, Damji KF, Freedman S, Moroi SE, Shafranov G, Shields MB. Shields: tratado de glaucoma. $5^{\mathfrak{a}}$ ed. Rio de Janeiro: Cultura Médica; 2008.

4. Phelps CD, Thompson HS, Ossoinig KC. The diagnosis and prognosis of atypical carotid-cavernous fistula (red-eyed shunt syndrome). Am J Ophthalmol. 1982; 93(4):423-36.

5. Grove AS Jr. The dural shunt syndrome. Pathophysiology and clinical course. Ophthalmology. 1984;91(1):31-44.

6. Barrow DL, Spector RH, Braun IF, Landman JA, Tindall SC, Tindall GT. Classification and treatment of spontaneous carotid-cavernous sinus fistulas. J Neurosurg. 1985;62(2):248-56.

7. Palestine AG, Younge BR, Piepgras DG. Visual prognosis in carotid-cavernous fistula. Arch Ophthalmol. 1981;99(9):1600-3.

8. Keltner JL, Gittinger JW Jr, Miller NR, Burder RM. A red eye and high intraocular pressure. Surv Ophthalmol. 1987;31(5):328-36.

9. Pierre Filho PT, Medina FM, Rodrigues FK, Carrera CR. Central retinal artery occlusion associated with traumatic carotid cavernous fistula: case report. Arq Bras Oftalmol. 2007;70(5):868-70.

10. Kupersmith MJ, Berenstein A, Choi IS, Warren F, Flamm E. Management of nontraumatic vascular shunts involving the cavernous sinus. Ophthalmology. 1988; 95(1):121-30.

11. Costa VP, Sampaio AP, Narimatsu C, Goldbaum M, Rodrigues-Alves CA. Fístulas carótido-cavernosas: descrição de quatro casos e revisão de literatura. Rev Bras Oftalmol. 1993;52(5):23-9.

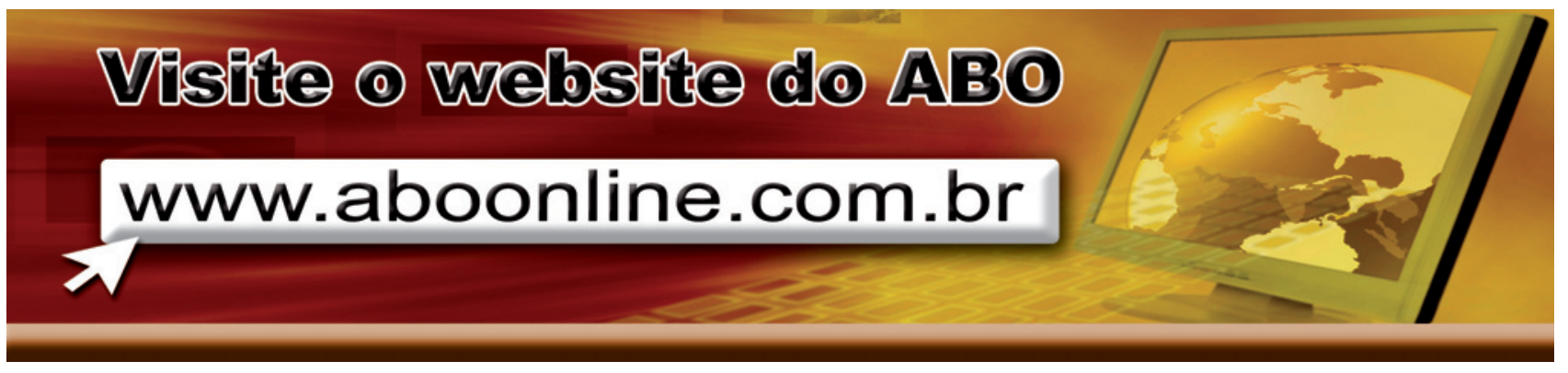

